\title{
Empatía y respeto entre otros valores: análisis de experiencias docentes de colaboración con organizaciones sociales
}

\author{
Melissa Andrea Flores Águila \\ Universidad de Magallanes, Punta Arenas, Chile. \\ http://orcid.org/0000-0001-6176-140X
}

Recibido: 19/05/202020 Revisado: 09/06/2020 Publicado: 22/06/2020

\begin{abstract}
Resumen
En este artículo se analizan cuatro experiencias de aprendizaje y servicio (ApS) desarrolladas entre los años 2016 y 2019 , con el objetivo de identificar las implicancias de esta estrategia educativa, en la formación valórica de futuros profesionales. A través de una metodología cualitativa, que incorporó como instrumentos de recolección, el análisis documental y grupos focales de discusión, se identificaron las percepciones de 3 estudiantes y 4 docentes de la carrera de Terapia Ocupacional que participaron de dichas experiencias. Se identifica que, en el contexto, es donde ocurre la consolidación de valores fundamentales para ejercer la futura profesión, siendo el agente comunitario un actor fundamental en el proceso formativo. La intervención territorial permitió fortalecer la promoción de valores como empatía, respeto, igualdad y equiparación de oportunidades. El proceso formativo cobra sentido si se realiza en colaboración con el contexto, identificando problemáticas y buscando soluciones para superarlas, generando así corresponsabilidad y ética social.

Palabras clave: Educación superior; responsabilidad social; valores sociales; igualdad de oportunidades.
\end{abstract}

\section{Empathy and respect among other values: analysis of collaborative teaching experiences with social organizations}

\begin{abstract}
In this article, four learning and service experiences (ApS) developed between 2016 and 2019 were analyzed, with the aim of identifying the implications of this educational strategy in the values growth of future professionals. Through a qualitative methodology, which incorporated documentary analysis and focus group discussions, the perceptions of students and academics from the Occupational Therapy program who participated in these experiences were identified. It is evident that, in the territory, it is where the enclave of fundamental values occurs to exercise the future profession, the community agent being a fundamental actor in the training process. The territorial intervention made it possible to strengthen the promotion of values such as empathy, respect, equality and equal opportunities. The training process makes sense if it is carried out in collaboration with the territory, identifying problems and seeking solutions to overcome them, thus generating co-responsibility and social ethics.
\end{abstract}

Keywords: Higher education; social responsability; social values; equal opportunities. 


\title{
Empatia e respeito entre outros valores: análise de experiências de ensino colaborativo com organizações Sociais
}

\begin{abstract}
Resumo
Neste artigo, são analisadas quatro experiências de aprendizagem e serviço (ApS) desenvolvidas entre 2016 e 2019, com o objetivo de identificar as implicações dessa estratégia educacional no valor da formação de futuros profissionais. Por meio de uma metodologia qualitativa, que incorporou a análise documental e as discussões em grupos focais como instrumentos de coleta, foram identificadas as percepções de alunos e acadêmicos do programa de Terapia Ocupacional que participaram dessas experiências. É evidente que, no território, é onde ocorre o enclave de valores fundamentais para o exercício da futura profissão, sendo o agente comunitário um ator fundamental no processo de formação. A intervenção territorial permitiu fortalecer a promoção de valores como empatia, respeito, igualdade e igualdade de oportunidades. 0 processo de treinamento faz sentido se for realizado em colaboração com o território, identificando problemas e buscando soluções para superá-los, gerando corresponsabilidade e ética social.
\end{abstract}

Palavras-chave: Educação superior; responsabilidade social; valores sociais; igualdade de oportunidades.

Citar como:

Flores, M. (2020). Empatía y respeto entre otros valores: análisis de experiencias docentes de colaboración con organizaciones sociales. Revista Digital de Investigación en Docencia Universitaria, 14(1), e1195. https://doi. org/10.19083/ridu.2020.1195

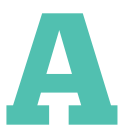
lo largo de este trabajo se analizaron experiencias de ApS en la carrera de Terapia Ocupacional, con el objetivo de identificar las implicancias de dichas experiencias en la formación valórica de los futuros profesionales y el rol de los agentes comunitarios en el proceso formativo de personas socialmente responsables.

Durante la primera década del siglo XXI, hubo una fuerte demanda social en países Iberoamericanos de un modelo de educación superior que aporte a la sociedad profesionales con sentido de responsabilidad, desarrollándose en profundidad el concepto de Responsabilidad Social Universitaria (RSU) (Martí \& Martí-Vilar, 2013). La responsabilidad, desde un enfoque ético, es la causa de la humanización, en el entendido que el ser humano es responsable, desde siempre, de todos y todo (Valleys, 2018). No basta con conocer el concepto de responsabilidad social para ejercer dicho valor, para que las personas adultas sean capaces de responder ante las necesidades de otros y tomar conciencia de su deber, tienen que ejercer conductas socialmente responsables (Navarro, 2013). En ese sentido, la educación puede ser considerada como un motor vertebrado del desarrollo humano y el desafío sería centrar la mirada en la calidad de la formación (Martí, Moncayo, \& Martí-Vilar, 2014).

Licando y Yepes (2018), señalan que el abandono de los componentes de formación humanística, particularmente en aquellos relacionados con la ética y los vínculos de integración social, es uno de los problemas de la función docente en la Educación Superior (ES). Una probable explicación al distanciamiento actual de las universidades con la dimensión simbólico-territorial del impacto de sus actividades es la escasa conversación de sus currículos o disciplinas con ella. Algunas disciplinas dialogan constantemente con su entorno, pero en general la formación en el territorio es incipiente en las universidades (Quinteros, 2019).

A pesar de ello, la evidencia demuestra que las universidades cuentan con herramientas pedagógicas que han fortalecido su misión formativa, $\mathrm{y}$ la transferencia del conocimiento generado en el aula, con el fin de disponer de instancias de diálogo con los agentes sociales, educativos y políticos del entorno que les rodea (Ruiz-Berajano \& Alastor, 2018). Una de esas estrategias es la metodología de Aprendizaje más Servicio (ApS), propuesta educativa que combina procesos de aprendizaje y de 
servicio a la comunidad, en un solo proyecto bien articulado, de forma que los estudiantes aprenden a trabajar en necesidades reales o problemáticas del entorno. Esta metodología combina el aprendizaje a través de la experiencia y acción de servicio a la comunidad (Rodríguez-Martín, 2017).

El aprendizaje y servicio supone una práctica educativa que favorece la adquisición de competencias en nuevos espacios docentes, al momento que da lugar al encuentro entre diferentes matices en una praxis inmersa en el mundo real, al prestar un servicio a los sectores más vulnerables de la sociedad (Simó, 2013). Es a partir de la psicoeducación sociomoral que se puede presentar una propuesta educativa para el contexto actual, planteándose vías de aprendizaje ético para que los estudiantes universitarios construyan y elaboren su autonomía moral, con la finalidad de alcanzar la madurez moral desde un aprendizaje ético basado en la autonomía, el diálogo y el respeto a uno mismo y al bien común (Martí, Martí-Vilar, \& Almerich, 2014).

Los valores morales son los pilares que sustentan los comportamientos y formas de vida de los seres humanos, no es una sociedad aislada, sino inserta en un mundo globalizado, pues los valores en la sociedad actual son fruto de los cambios económicos y sociales que los transforman profundamente. (Vera et al., 2016). Es por esta razón que resulta necesario analizar los procesos de formación integral en la educación superior, y el impacto que tiene la relación con el medio en la adquisición de competencias profesionales y valóricas.

\section{Marco teórico}

La ética en el ejercicio profesional es el garante de un desempeño honesto y conducta socialmente responsable, sobre todo de aquel que debe brindar su servicio a otras personas (Salazar, Salazar, Rodríguez, \& Díaz, 2019). En el caso de los profesionales de la salud, por ejemplo, la calidad de su formación va a depender en gran medida de la construcción de sus propios valores para regular su actuar profesional futuro, a partir de los cuales, se rija su conducta ética, moral y humanística. La calidad en la formación profesional, no depende solamente de los conocimientos y habilidades que desarrolle el currículo académico, sino también de aquellos intereses y valores que regulen su ejerci- cio profesional (Sosa et al., 2016).

Por lo tanto, la formación profesional debe ir de la mano de la educación moral y valórica, siendo parte del papel de la Educación Superior, el aprendizaje de la autonomía moral, el respeto a sí mismo y la búsqueda del bien común. Para Martí, Martí- Vilar y Almerich (2014), dicha optimización beneficiaría las áreas cognitivo-racionales (razonamiento moral, juicio moral y toma de decisiones); área emocional-afectiva (sensibilidad moral y experiencias sociomorales) y motivacional-conductual (esfuerzo y regulación de la conducta prosocial). Es por ello relevante, profundizar en la formación como persona socialmente responsable, con conciencia ciudadana y conductas prosociales que están a la base de su quehacer. La formación de ciudadanía activa, implica propiciar espacios de participación social-comunitaria, que permitan aportar en mejorar la calidad de vida de un contexto vulnerable (Briede \& Mora, 2016). No basta con una educación superior de calidad si esa calidad no contempla una formación valórica integral y humanitaria. Las instituciones de educación superior deben ser capaces de dar respuesta a la demanda de la Organización de las Naciones Unidas para la Educación, la Ciencia y la Cultura (Unesco), en relación a la necesidad de formar profesionales con una sólida preparación, junto a una comprensión de las necesidades del entorno que con sus conocimientos pueden apoyar a resolver (Martí, Martí-Vilar, \& Puerta, 2011).

En este punto es necesario señalar que la formación valórica no es lineal ni mecánica, sino más bien, corresponde a una elaboración personal en virtud de la cual, los seres humanos en interacción con el medio, histórico-social en el que se desenvuelven, desarrollan sus propios valores, y en este sentido las instituciones educativas tienen un destacado rol para propiciar que dicha interacción se lleve a cabo (Trujillo, 2016). Para el aprendizaje de la autonomía moral, el respeto y el bien común y el diálogo, la optimización sería una acción en tres áreas: en primer lugar, las áreas cognitivo-racionales, relacionado con el razonamiento y juicio moral y la toma de decisiones; área emocional-afectiva, que considera la sensibilidad moral y experiencias sociomorales y finalmente el área motivacional-conductual, que implica el esfuerzo y regulación de la conducta prosocial (Martí-Vilar, 
Vargas, Moncayo, \& Martí, 2014).

En este sentido, los proyectos de ApS pueden ser una estrategia válida para el proceso de formación valórica de los futuros profesionales, debido a que permiten ejercer razonamiento clínico, toma de decisiones, conexión emocional con las personas y sus necesidades y generar conductas impulsadas por su motivación de generar cambios. Estas experiencias se conectan, con la dimensión ética de la educación superior, necesaria para dar respuesta a los problemas actuales e históricos de la comunidad, y para favorecer la formación integral de los futuros profesionales (Ruiz-Berajano \& Alastor, 2018).

El ApS permite a los estudiantes conectarse con un escenario comunitario, en el que movilizarán saberes tanto disciplinarios, como valóricos y sellos institucionales de su formación, con el fin de que les permitan otorgar soluciones para satisfacer necesidades y mejorar la calidad de vida de la población. De la misma manera, se presentan objetivos de aprendizaje que los estudiantes alcanzarán desarrollando esa acción solidaria y activa (Briede \& Mora, 2016). El estilo educativo del ApS concede el protagonismo pedagógico de cada experiencia al estudiante, y este va desarrollando un proceso de reconocimiento del otro, en la medida en que se conectan con las personas que forman parte de comunidades vulnerables, dicho reconocimiento implica valorar al otro y ofrecerle afecto, derechos y estima (García-Pérez \& Mendía, 2015; Puig \& Bär, 2016).

Para docentes y estudiantes del área de la salud, los valores profesionales tienen como base valores personales de carácter moral y valores éticos inherentes al quehacer profesional, donde destacan, identidad y vocación profesional, responsabilidad, veracidad, respeto, amistad, justicia, y conocimiento científico (Vera et al., 2016). La identificación de los valores esenciales en un profesional de la salud y la incorporación de estos por las nuevas generaciones, requieren del diseño y aplicación de métodos eficaces que permitan conocer cuáles son los valores estimados, hasta qué punto pueden estos orientar hacia los fines de cada profesión y cómo pueden ser incorporados a lo largo de la formación universitaria (Véliz, Döner, Gonzáles, \& Ripoll, 2017).

Como una primera aproximación a identificar los valores de los terapeutas ocupacionales en for- mación y la implicancia de los agentes comunitarios, se utilizó en este estudio, una metodología de tipo cualitativa, descriptiva y transversal, ya que, según Hernández, Fernández y Baptista (2006), este tipo de estudio permite detallar situaciones y eventos. Es decir, cómo es y cómo se manifiesta determinado fenómeno y busca especificar propiedades importantes de personas, grupos y comunidades. Los estudios cualitativos sobre el desarrollo de los valores declarados en el modelo profesional en una carrera, constituyen una herramienta de sumo valor para mejorar las acciones educativas en una institución y conocer el funcionamiento de las estrategias metodológicas utilizadas, aportará a organizar la labor docente en su función de educar en valores a los futuros profesionales (Trujillo, 2016).

Se utilizó como mecanismo de recolección de información la revisión de datos secundarios, que corresponden a informes y documentos relacionados con la ejecución de las Experiencias de ApS, desarrolladas entre 2016 y 2019 en la Comuna de Punta Arenas, de la Región de Magallanes, Chile. Asimismo, se realizaron entrevistas semiestructuradas y grupos focales con estudiantes y docentes.

La unidad de análisis fue, por una parte, el texto extraído de los documentos y, por otra parte, el análisis del discurso de los participantes. Los informantes primarios fueron personas que formaron parte de las experiencias: 4 docentes y 3 estudiantes. El procedimiento de muestreo fue intencionado, considerando el nivel de implicancia con las experiencias desarrolladas.

A continuación, y a modo de contextualización, se presentan las experiencias de ApS a partir de las agrupaciones que fueron socias comunitarias del proceso formativo y las estrategias utilizadas en conjunto con la carrera (Ver figura 1).

Los proyectos desarrollados en colaboración con organizaciones comunitarias, en este caso, agrupaciones sociales de y para personas con discapacidad, han buscado intercalar las funciones intrínsecamente docentes, con la formación de una ciudadanía socialmente comprometida y solidaria (Vallaeys, 2008). Cabe destacar que el análisis en esta oportunidad no estuvo centrado en el desarrollo de las experiencias, ni en su impacto territorial, y tampoco en las competencias profesionales adquiridas por los estudiantes, sino 


\begin{tabular}{|c|c|c|c|c|}
\hline & REPRODIS & YAFUN & AGACI & SENADIS \\
\hline $\begin{array}{l}\text { Descripción de } \\
\text { la organización }\end{array}$ & $\begin{array}{l}\text { - Organización } \\
\text { comunitaria que } \\
\text { busca generar } \\
\text { progreso social, } \\
\text { erradicar barreras, } \\
\text { equiparar } \\
\text { oportunidades e } \\
\text { influir como agente } \\
\text { de cambio dentro } \\
\text { de la sociedad. }\end{array}$ & $\begin{array}{l}\text { - Agrupación de } \\
\text { amigos y pacientes } \\
\text { con artritis } \\
\text { reumatoide, que } \\
\text { busca alternativas } \\
\text { oportunas de } \\
\text { tratamiento y } \\
\text { mantención de } \\
\text { roles e inclusión } \\
\text { social. }\end{array}$ & $\begin{array}{l}\text { - Organización } \\
\text { compuesta por } \\
\text { familiares, amigos } \\
\text { y personas con } \\
\text { discapacidad } \\
\text { visual. Desde hace } \\
30 \text { años trabaja por } \\
\text { la inclusión social } \\
\text { y laboral de las } \\
\text { personas ciegas. }\end{array}$ & $\begin{array}{l}\text { Servicio público } \\
\text { que tiene por } \\
\text { finalidad promover } \\
\text { el derecho a } \\
\text { la igualdad de } \\
\text { oportunidades } \\
\text { de las personas } \\
\text { con discapacidad, } \\
\text { a través de la } \\
\text { coordinación del } \\
\text { accionar del Estado. }\end{array}$ \\
\hline $\begin{array}{l}\text { Objetivo de la } \\
\text { experiencia Aps. }\end{array}$ & $\begin{array}{l}\text { Promover y } \\
\text { resguardar los } \\
\text { Derechos Humanos } \\
\text { de las personas } \\
\text { en situación de } \\
\text { vulnerabilidad } \\
\text { social. }\end{array}$ & $\begin{array}{l}\text { - Promover la } \\
\text { inclusión, y } \\
\text { desarrollo de } \\
\text { actividades } \\
\text { orientadas } \\
\text { a mejorar su } \\
\text { bienestar y } \\
\text { calidad de vida } \\
\text { para obtener una } \\
\text { participación } \\
\text { social activa en la } \\
\text { comunidad. }\end{array}$ & $\begin{array}{l}\text { Propender la } \\
\text { participación, } \\
\text { inclusión social } \\
\text { y rehabilitación } \\
\text { de las personas } \\
\text { con discapacidad } \\
\text { sensorial visual. }\end{array}$ & $\begin{array}{l}\text { Promover el } \\
\text { derecho a la } \\
\text { igualdad de } \\
\text { oportunidades de } \\
\text { las personas con } \\
\text { discapacidad, con } \\
\text { el fin de obtener } \\
\text { su inclusión social, } \\
\text { contribuyendo al } \\
\text { pleno disfrute de } \\
\text { sus derechos. }\end{array}$ \\
\hline $\begin{array}{l}\text { Estrategias de } \\
\text { intervención en } \\
\text { conjunto con la } \\
\text { Carrera }\end{array}$ & $\begin{array}{l}\text { - Capacitación y } \\
\text { educación a PcD } \\
\text { y sus familias } \\
\text { en relación a } \\
\text { derechos sexuales y } \\
\text { reproductivos. } \\
\text { - Asesoría de } \\
\text { profesionales } \\
\text { e instituciones } \\
\text { en relación a } \\
\text { los derechos de } \\
\text { maternidad y } \\
\text { paternidad de PcD. } \\
\text { Apoyo en casos } \\
\text { individuales de PcD. }\end{array}$ & $\begin{array}{l}\text { - Generación de } \\
\text { un taller de } \\
\text { intervención } \\
\text { terapéutica grupal. } \\
\text { - Atenciones } \\
\text { individuales en } \\
\text { la universidad y a } \\
\text { domicilio. } \\
\text { - Diseño y confección } \\
\text { de órtesis y ayudas } \\
\text { técnicas. } \\
\text { - Capacitaciones a } \\
\text { miembros de la } \\
\text { organización y } \\
\text { otras instituciones } \\
\text { del territorio. }\end{array}$ & $\begin{array}{l}\text { - Diseño y ejecución } \\
\text { de un proyecto de } \\
\text { "turismo accesible", } \\
\text { específicamente } \\
\text { la experiencia } \\
\text { de navegación } \\
\text { en Kayak por } \\
\text { el estrecho de } \\
\text { Magallanes. } \\
\text { - Realización de } \\
\text { seminario de } \\
\text { Turismo accesible } \\
\text { - Capacitación a } \\
\text { operadores y guías } \\
\text { turísticos de la } \\
\text { región. }\end{array}$ & $\begin{array}{l}\text { Ejecución de } \\
\text { la estrategia de } \\
\text { Desarrollo Local } \\
\text { inclusivo, desde los } \\
\text { gobiernos locales, } \\
\text { en alianza con las } \\
\text { organizaciones } \\
\text { comunitarias. } \\
\text { - Desarrollo de } \\
\text { escuelas de gestión } \\
\text { y liderazgo para } \\
\text { organizaciones de } \\
\text { PcD. }\end{array}$ \\
\hline
\end{tabular}

Figura 1. Socios comunitarios y estrategias utilizadas.

en la formación valórica y el rol de los agentes comunitarios en el proceso formativo de los futuros profesionales.

\section{Proceso de formación valórica a lo largo de la carrera}

Es importante evidenciar en los futuros profesionales de salud cómo se va configurando el repertorio valórico y cómo la formación profesional incide en los valores personales y sociales con los que los estudiantes acceden a la educación superior (Véliz, Döner, Gonzáles, \& Ripoll, 2017). Docentes y estudiantes, que participaron de las experiencias descritas en este trabajo, concuerdan en que existen momentos clave a lo largo de la formación valórica. En primer lugar, mencionan el momen- to que se da en el aula, a través de la transmisión de experiencias de los docentes y los procesos reflexivos. Los estudiantes consideran relevante poder acercarse a las vivencias y significados de sus docentes, a través del análisis de estudios de casos clínicos, ejemplos, videos, literatura, entre otros. Esto se relaciona con el área cognitivo emocional, ya que está estrechamente vinculado al razonamiento y juicio moral. Otro elemento del proceso de formación valórica o moral, es el acercamiento a la realidad a través de la vinculación con su entorno social, momento en el que los estudiantes comienzan los cuestionamientos y el enfrentamiento con la realidad, es ahí donde ellos pueden poner en práctica valores como la empatía, el respeto, la confidencialidad. El enfrentamiento 


\begin{tabular}{|c|c|}
\hline Visión & Testimonio (hallazgo) \\
\hline $\begin{array}{l}\text { Visión de } \\
\text { docentes }\end{array}$ & $\begin{array}{l}\text { "La formación valórica de los estudiantes se realiza en dos niveles. En primer lugar, en el aula, } \\
\text { acercándolos a la realidad y sus necesidades a través de análisis de casos, ejemplos, videos, lecturas, } \\
\text { analizando en profundidad el quehacer desde lo técnico y profesional pero también desde su actuar } \\
\text { valórico." (D.3) } \\
\text { “Es importante considerar que no partimos con una hoja en blanco, nuestros estudiantes vienen con } \\
\text { un bagaje valórico previo, eso facilita el proceso de ir construyendo competencias profesionales y } \\
\text { valóricas hasta convertirse en un profesional con responsabilidad social. (D.3) } \\
\text { “Los valores que traen los estudiantes son reforzados con la mirada humanista de la Terapia } \\
\text { ocupacional...En ocasiones es más consciente, en otras surge desde nuestras experiencias, el ir } \\
\text { transmitiendo a los estudiantes una forma de ver al otro como personas que merecen nuestro respeto } \\
\text { y empatía, cómo vivir en armonía con los demás...Es un proceso de deconstrucción de los valores que } \\
\text { traen consigo y hacer una nueva construcción individual y colectiva. (D.1) }\end{array}$ \\
\hline $\begin{array}{l}\text { Visión de } \\
\text { estudiantes }\end{array}$ & $\begin{array}{l}\text { “Traemos valores desde antes de entrar a la universidad, pero a lo largo de la carrera nos refuerzan valores } \\
\text { que nos permitirán relacionarnos adecuadamente con nuestros usuarios y con la comunidad." (E. 2) } \\
\text { “A lo largo de la carrera nos muestran una forma de vivir esta disciplina, y sus experiencias, tomar lo } \\
\text { positivo. Eso es un proceso formativo, ser mejor más adelante, y lo que nos relatan los docentes nos } \\
\text { permiten identificar cómo debemos actuar y relacionarnos con otros." (E.1) } \\
\text { "Nuestros docentes nos hacen ver otras realidades a través de sus experiencias, pero también } \\
\text { acercándonos a la realidad. Ahí es cuando comienzan los cuestionamientos y a poner en práctica } \\
\text { valores como la empatía, respeto, confidencialidad, etc. Al estar con otras personas y sus realidades, } \\
\text { distintas a veces a las nuestras." (E.2) } \\
\text { "Creo que la formación ética no basta con aspectos teóricos, es necesario poner en práctica } \\
\text { con las personas de la comunidad. Ellos nos hacen ver lo que necesitan de nosotros y también } \\
\text { comprendemos lo que somos capaces de entregar".(E.3) }\end{array}$ \\
\hline
\end{tabular}

Figura 2. Proceso de formación valórica en Terapia Ocupacional.

con una realidad que no conocían, o que habían visto de lejos, genera una acción en el área emocional-afectiva, de la que habla Martí - Vilar et ál. (2014). Los estudiantes refieren que al acercarse a la realidad pueden identificar las necesidades de las personas y de esa manera proponer cómo podrían apoyarlos, colaborar en la satisfacción de dichas necesidades, es decir, pasar de la emoción a la acción. Su interés de movilizarse y generar un cambio positivo en esa realidad, sería una acción en el área motivacional-conductual, lo que generaría finalmente la conducta prosocial.

En la figura 2, se aprecia que la formación valórica es un proceso de co-construcción, que parte en el aula y se fortalece en el territorio, docentes, compañeros y agentes sociales refuerzan el bagaje valórico que viene desde el hogar y se adquieren competencias éticas propias del quehacer profesional.

Un espacio para la reflexión sobre principios como la justicia social, los derechos humanos o la ciudadanía, sería fundamental para favorecer el aprendizaje sobre su propia capacidad de mejorar la situación actual (Simó, Ginesta, \& San Eugenio,
2013). Términos como sostenibilidad, derechos humanos, igualdad, gestión de la diversidad, son la expresión social de un deseo interno de desarrollo humano en base al contexto social, la capacidad para sentir como propias y vitales estas concepciones llevaría a la persona a ser responsable en base a la regulación emocional (Martí-Vilar, et ál., 2011). La implicación de los estudiantes en las actividades desarrolladas en un contexto social, les permite mostrar habilidades que a menudo no pueden desarrollar en el currículo oficial, y sentir que hacen un aporte socialmente útil durante su experiencia educativa. El acompañamiento educativo es una dimensión fundamental en los procesos de crecimiento y desarrollo de individuos y grupos, en este contexto, y tal como lo mencionan los estudiantes entrevistados, el docente, es un actor clave en la articulación de la experiencia que favorece la implicancia de los estudiantes en su comunidad, lo que constituye una base relacional y socioafectiva para el trabajo de formación valórica y ciudadana (García-Pérez \& Mendía, 2015; Jara, Sánchez, \& Cox, 2019) 


\section{Aporte de los agentes comunitarios en el proceso formativo valórico del estudiante.}

La metodología Aprendizaje más Servicio, se ha presentado como una herramienta que permite responder a la necesidad de acercamiento de la formación profesional a la realidad contextual. Permite abarcar tres dimensiones: la académica, donde el contacto directo con la realidad posibilitaría mayores y mejores aprendizajes en los estudiantes; otra ligada con la calidad del servicio y a la problemática comunitaria; y finalmente, la posibilidad de generar un espacio de formación en valores para los estudiantes, estructurados de reflexión, relacionando el conocimiento y el servicio (Jouannet, Salas, \& Contreras, 2013).

Estudiantes y docentes que fueron parte de las experiencias territoriales presentadas en este artículo, pusieron énfasis en la relevancia que tenían los socios comunitarios, como un actor clave en el proceso formativo moral y valórico de los futuros profesionales. El intercambio de estas experiencias, permiten internalizar aspectos morales como la cooperación y respeto mutuo, donde, junto con enfrentar la toma de decisiones durante su desarrollo académico, surge la preocupación por otros, por una comunidad con la que van a trabajar a lo largo de su ejercicio profesional, exigiendo un compromiso y determinación de objetivos personales que se encuentren en la misma dirección de los objetivos de la sociedad (Bustamante \& Navarro, 2007).

En la figura 3, se observa que el aporte de los socios comunitarios es ampliamente apreciado por estudiantes y docentes en relación a la retroalimentación que entregan al futuro profesional para su crecimiento valórico y formación integral.Las organizaciones sociales que han formado parte de estas experiencias, han sido capaces de entender su rol formativo y asumido la misión de apoyar el fortalecimiento del recurso humano que llega a prestar sus servicios. Ellos valoran este

\begin{tabular}{|c|c|}
\hline Visión & Testimonio (hallazgo) \\
\hline $\begin{array}{l}\text { Visión de los } \\
\text { docentes }\end{array}$ & $\begin{array}{l}\text { “La relación con el socio comunitario es fundamental para formar a nuestros estudiantes, } \\
\text { aprendemos de ellos y con ellos... nos incentiva a seguir avanzando como profesionales y como } \\
\text { personas.” (D.2) } \\
\text { “Al tener relación con organizaciones de la comunidad y a portar a la satisfacción de sus necesidades, } \\
\text { los estudiantes también van empoderándose y aumentando su autoestima. Y entienden la } \\
\text { importancia de su rol profesional."(D.4) } \\
\text { “Existe una necesidad y reciprocidad en el trabajo colaborativo. La sociedad civil con la que trabajamos } \\
\text { da valor especial a la disciplina en pro del cumplimento de sus objetivos y para el desarrollo local...No } \\
\text { visualizo una formación valórica en Terapia Ocupacional sin redes comunitarias.” (D.3) } \\
\text { “Nuestros socios comunitarios tienen un gran rol de retroalimentación activa y constante, tanto a los } \\
\text { docentes como a los estudiantes...en la informalidad de su relación diaria van reforzándoles valores } \\
\text { y haciéndoles ver cuando estos no están presentes. Son activos y claros en decir qué calidad de } \\
\text { profesionales quieren y necesitan. La comunidad es protagonista de los procesos de aprendizajes y } \\
\text { formación valórica, no podríamos hacerlos sin los socios comunitarios.”(D.1) }\end{array}$ \\
\hline $\begin{array}{l}\text { Visión de los } \\
\text { estudiantes }\end{array}$ & $\begin{array}{l}\text { “Para mí las organizaciones con las que hemos trabajado tienen también un rol de educadores, para } \\
\text { mí fue un desafío enfrentarme a esa nueva realidad y ellos me apoyaron y enseñaron a relacionarme } \\
\text { con las personas." (E.1) } \\
\text { "Al poder insertarnos en un colectivo social podemos ver la realidad y las experiencias de otros, } \\
\text { podemos comprender las situaciones y vivencias que ellos han tenido que enfrentar. Ejercemos una } \\
\text { interdependencia, trabajamos en conjunto con la comunidad por una meta y objetivo común. Vamos } \\
\text { construyendo valores, además de los aprendizajes del aula." (E.3) } \\
\text { “Sin la experiencia que viví con Reprodis, por ejemplo, siento que jamás habría sido capaz de } \\
\text { relacionarme adecuadamente con las personas, no solo desde lo profesional, sino de los valores } \\
\text { personales que se necesitan para nuestra profesión." (E.2) } \\
\text { "Los aspectos éticos y valóricos se han reforzado desde la vinculación con el medio, considerando al } \\
\text { usuario desde un aspecto integral, abarcando su ambiente y sus redes, familias, amigos...conociendo } \\
\text { otras realidades se refuerzan esos valores".(E.1) }\end{array}$ \\
\hline
\end{tabular}

Figura 3. Aporte de los socios comunitarios en el proceso formativo integral 
recurso humano joven y esperanzador, no sólo como una mano de obra gratuita y profesional, con la que de otro modo no podrían contar, sino también les transmiten que en sus manos está el futuro de la sociedad (Echeverría-Falla, 2013). Del mismo modo, como refieren estudiantes y docentes, la retroalimentación entregada por el socio comunitario es honesta y desinteresada, diariamente refuerzan aquellos valores positivos que se evidencian en la interacción o solicitan cambio de actitudes o compromiso cuando notan que ese aspecto esta deficitario. Cuando el socio comunitario entiende su rol y no se enfrenta al estudiante como un sujeto de beneficio o caridad, sino que exige un servicio de calidad y con respeto, se logran cambios importantes e imborrables en el futuro profesional.

\section{Valores relacionados con el futuro ejercicio profesional y que se fortalecen en las experiencias ApS.}

A lo largo del diálogo con los participantes, se pudieron identificar los valores fortalecidos en las experiencias ApS para desempeñar adecuadamente el rol profesional. Rol que está marcado por un empoderamiento de las personas con las que se trabaja, a través de la educación, la asociatividad y participación social, la organización y la comunicación social (Flores, Contreras, Levicoi, \& Vargas, 2015). El Código de ética del Colegio de Terapeutas Ocupacionales de Chile, plantea que este profesional deberá tener en sus competencias un profundo sentido de la moral de manera que ponga su quehacer al servicio de la sociedad e impulse su progreso y bienestar, procurando actualizar y perfeccionar sus conocimientos para cooperar al desarrollo de la ciencia y de las técnicas de su profesión.

En las discusiones de grupo, los participantes refirieron:

- "Valores como el respeto y empatía, son fundamentales porque necesitamos visualizar y reconocer al otro como sujeto y titular de derecho" (D.3)

- "Los Terapeutas Ocupacionales trabajamos con personas muy vulnerables, quizás las más vulnerables y vulneradas de la sociedad, por lo tanto, el componente valórico es tan importante como las competencias como profesionales...debemos luchar para que esas personas nunca más sean vulneradas" (D.1)

- "La ética y la empatía, el respeto, la confidencialidad son valores que tenemos que tener con nuestros usuarios... y con ellos luchar por la igualdad y la equidad, que son también valores sociales importantes en nuestro trabajo como futuros TO." (E.1)

- "La formación valórica es fundamental puesto que vamos a trabajar con y para las personas." (E.2)

Cuando los estudiantes adquieren competencias valóricas no sólo se están formando para una ciudadanía comprometida, le están encontrando un sentido a su propia existencia. Los valores nos ayudan a guiar en nuestra vida la adquisición y fortalecimiento de la prosocialidad. Esto les permitirá a los profesionales trabajar en conjunto con sus comunidades por alcanzar la igualdad y equiparación de oportunidades, el ejercicio y reivindicación de los derechos humanos (Simó, 2013).

Cuando una persona dedica su ejercicio profesional a acompañar a personas que han sido vulneradas en sus derechos, y han sufrido exclusión social, debe indudablemente tener un bagaje valórico que le permita comprender plenamente las necesidades del otro, situarse desde su validación como ser humano pleno de derechos, y ponerse al servicio de éste, acompañándolo en su lucha por la rehabilitación y la reinserción social. Una de las funciones educativas de las universidades es precisamente orientar el currículo y las formas de enseñanza a la realidad del país, ofrecer a los estudiantes oportunidades de acercamiento a la realidad social y de servicio, generar conciencia de las implicaciones éticas de su actuación personal y profesional (AUSJAL, 2009, como se citó en Licando \& Yepes, 2018).

\section{Discusión:}

El objetivo de analizar las experiencias docentes, fue identificar las implicancias del ApS en la formación valórica de los futuros profesionales. Los resultados obtenidos muestran, que estudiantes y docentes consideran las experiencias de intervención territorial, como parte fundamental del proceso de formación valórica. Reconociendo que 
es ahí, en el territorio, donde ocurre el enclave de valores fundamentales para ejercer su futura profesión. Las experiencias de Aps permiten en un contexto real, que docentes, estudiantes y comunidad, trabajen colaborativamente en la ejecución de acciones que cambien la realidad social, en este caso, de grupos socialmente excluidos y vulnerados en sus derechos de participación social.

Se pudo evidenciar una vez más, que la educación superior posee las herramientas para generar un conocimiento transformador desde la propia realidad. Formando profesionales al mismo tiempo que entrega un servicio a la sociedad, en especial a los colectivos más frágiles y vulnerables, consiguiendo una doble ganancia: social y académica (Simó, 2013). La educación superior tiene un rol preponderante en formación de profesionales socialmente responsables, por lo que debe garantizar espacios participativos en la producción y trasferencia de conocimientos, para generar un cambio moral e ideológico de personas en cuyas manos estará el desarrollo de la sociedad (Alcaín \& Medina-García, 2017).

En los últimos años, investigaciones que analizan experiencias de Aps concluyen que, a través de esta estrategia de enseñanza, se intenciona la formación de ciudadanía activa, la responsabilidad social, el trabajo colaborativo y la capacidad para comunicar ideas. En la medida en que se propicia la articulación de los saberes teóricos-prácticos disciplinarios, en un contexto situado, se desarrollan valores que humanizan a los estudiantes, necesarios para el desarrollo social profesional y personal. Las investigaciones analizadas, hacen énfasis en la adquisición de valores como responsabilidad individual y social, respeto, tolerancia, solidaridad, generosidad, empatía, colaboración, entre otros y competencias profesionales como la comunicación y la planificación de actividades. (Briede \& Mora, M, 2016; García \& Sánchez, 2017; Montesi \& Villaseñor, 2018; Salazar, Salazar, M., Rodríguez, C., \& Díaz, J., 2019)

Algunos aspectos que cabe destacar entre los elementos que surgen de este estudio, son el reconocimiento y la legitimidad, tanto a nivel personal como territorial. En los discursos de los participantes se repite que las experiencias de ApS les permitieron identificar al otro como un sujeto de derecho y por lo tanto merecedor de respeto y empatía, y también, como participe y colaborador del proceso formativo individual. Las acciones que involucran colaboración y participación activa de los estudiantes con los miembros de su comunidad, desencadenan en primer lugar que dicha comunidad entregue la licencia social/territorial que la autoriza a la universidad, a través de sus docentes y estudiantes a apoyarlos en la búsqueda de soluciones a sus necesidades $\mathrm{y}$, en segundo lugar, se genera un agradecimiento y valoración del servicio prestado (Puig \& Bär, 2016; Quinteros, 2019). Lográndose así el reconocimiento y legitimidad en un doble sentido desde la comunidad hacia el estudiante y desde este hacia las personas que forman parte de su territorio. La retroalimentación entregada en el trabajo diario permite al futuro profesional identificar carencias y necesidades sociales, y ser proactivo en la resolución de estas, encontrando su sitio e identificándose como un agente de cambio social. Para docentes y estudiantes todo cobra sentido cuando pasan de la razón a la emoción, y del sentimiento a la acción, este último paso fundamental para el logro de conductas prosociales (Martí, Moncayo, \& Martí-Vilar, 2014). De esa forma, la alianza con las organizaciones y colectivos sociales de los territorios se constituye, para la universidad, en la posibilidad de concreción de una vinculación con el medio externo que colabore efectivamente con los procesos socioculturales que producen en la realidad social, a través de la generación y rescate de conocimientos y saberes de la sociedad civil (Ketterer, Solá, Tragolaf, \& Obando, 2019).

Este estudio, si bien logró su objetivo de identificar aspectos de la formación valórica en las experiencias de ApS y el rol de los agentes comunitarios en este proceso, presenta algunas limitaciones necesarias de analizar. En primer lugar, el número de participantes es escaso, lo que permite describir una realidad puntual pero no podría ser considerado para extrapolar los resultados a la formación de terapeutas ocupacionales a un nivel más amplio. Por tal motivo, se recomienda la realización de estudios con mayor nivel de participación de informantes, dentro de los cuales se podría incorporar la colaboración entre distintas universidades. En segundo lugar, se contó con la participación de estudiantes y docentes, pero habría sido interesante poder conocer la opinión de 
los actores sociales que fueron protagonistas de estas experiencias, para identificar la percepción de su rol como agentes educativos y de esa manera triangular la información con todos los actores de las experiencias.

Las conclusiones de este trabajo y de otros estudios analizados, permiten evidenciar que resulta relevante continuar con procesos investigativos en esta temática. Se sugiere la profundización en elementos tales como: componentes valóricos en los perfiles ingreso de los estudiantes; propuestas metodológica orientadas a la formación de profesionales socialmente responsables; herramientas y competencias docentes para acompañar el proceso formativo valórico de los futuros profesionales y autonomía y proactividad de los estudiantes en las intervenciones territoriales, entre otras investigaciones que fortalezcan el conocimiento en la formación integral de los estudiantes en educación superior (Véliz, Döner, Gonzáles, \& Ripoll, 2017; Martí, Moncayo, \& Martí-Vilar , 2014; Sosa et al., 2016; García-Pérez \& Mendía, 2015).

Finalmente, se concluye que estudiantes y docentes identifican que el agente social es un elemento clave de retroalimentación constante en este proceso. Es importante resaltar que se pueden canalizar los esfuerzos docentes hacia la construcción de una comunidad más justa y solidaria, y desde las instituciones de educación superior acelerar los procesos de cambio social. La adecuada implantación del ApS en el contexto educativo desde la colaboración de Universidad-Centros educativos-Comunidad exige la implicación e impulso de la Administración apoyando esta red de trabajo colaborativo; la difusión de ideas, la presentación de las propuestas que han tenido éxito y la ayuda a la coordinación entre instituciones. El éxito en la implantación y el desarrollo del ApS parece inseparable del trabajo en equipo emprendido entre diferentes instituciones (Rodríguez-Martín, 2017). Si se comprende profundamente la educación como un bien común, resultará evidente el rol que todos forman en el proceso formativo de los futuros profesionales, en el recurso humano socialmente responsable está el futuro de una sociedad más justa y equitativa.

\section{Referencias}

Alcaín, E., \& Medina-García, M. (2017). Hacia una educación universitaria inclusiva: realidad y retos. Revista $D i$ gital de Ivestigación en Docencia Universitaria, 4-19. https://doi.org/10.19083/ridu.11.530

Briede, J., \& Mora, M. (2016). Diseño y Co-creación mediante aprendizaje y servicio en contexto vulnerable: Análisis de percepción de la experiencia. Formación Universitaria, 9(1), 57-70. https://doi.org/10.4067/ S0718-50062016000100007

Bustamante, M., \& Navarro, G. (2007). Auto-atribución de comportamientos socialmente responsables de estudiantes de carreras del área de ciencias sociales. Revistas perspectivas, 45-63. Recuperado de http://biblioteca-digital.ucsh.cl/greenstone/collect/revistal_old/ archiviers/HASH0142/4cefbc48.dir/Auto\%20atribuicion\%20de\%20comportamientos.pdf

Echeverría-Falla, C. (2013). Educación ética: ¿Normas o virtudes?¿Qué giro debe tomar la enseñanza en la formación de universitarios solidarios? Persona y Bioética, 17(2), 151-167. https://doi.org/10.5294/ pebi.2013.17.2.1

Flores, M., Contreras, C., Levicoi, Y., \& Vargas, C. (2015). Ocupación e identidad social de personas en situación de calle de la ciudad de Punta Arenas. Revista Chilena de Terapia Ocupacional, 15(2). https://doi. org/10.5354/0719-5346.2015.38159

García, M., \& Sánchez, L. (2017). El aprendizaje servicio y el desarrollo de las competencias emocionales en la formación inicial del profesorado. Contextos educa tivos(20), 127-145. https://doi.org/10.18172/con.2991

García-Pérez, A., \& Mendía, R. (2015). Acompañamiento educativo: El rol del educador en Aprendizaje y servicio Solidario. Profesorado. Revista de Currículum y Formación de profesorado, 19(1), 42-58. Recuperado el 17 de Enero de 2020, de https://www.redalyc.org/ articulo.oa?id=567/56738729004

Hernández, R., Fernández, C., \& Baptista, P. (2006). Metodología de la investigación, Cuarta edición. Mexico: McGraw-Hill Interamericana.

Jara, C., Sánchez, M., \& Cox, C. (2019). Liderazgo educativo y formación ciudadana: visiones y prácticas de los actores. Calidad en la Educación, 350-381. https://doi. org/10.31619/caledu.n51.687 
Jouannet, C., Salas, M., \& Contreras, M. (2013). Modelo de implementación de Aprendizaje servicio $(A+S)$ en la UC: Una experiencia que impacta positivamente en la formación profesional integral. Calidad en la educación, 39, 197-212. https://doi.org/10.4067/ S0718-45652013000200007

Ketterer, R., Solá, M., Tragolaf, A., \& Obando, C. (2019). Universidad y territorio: Vinculación con el medio como oportunidad de promover los derechos de las mujeres. El observatorio de género como desafío institucional. Revista GeoNordeste (2), 7-22.

Licando, O., \& Yepes, S. (2018). La Educación Superior conceptualizada como bien común: el desafío propuesto por Unesco. Revista Digital de Investigación en Docencia Universitaria, 12(1), 6-33. https://doi. org/10.19083/ridu.12.715

Martí, J., \& Martí-Vilar, M. (2013). Una década de responsabilidad social universitaria en Iberoamérica. Revista española del tercer sector, 25, 145-162. Recuperado de https://revistas.upcedu.pe/indexphp/docencia/ article/download/364/350

Martí, J., Martí-Vilar, M, \& Puerta, I. (2011). Hacia un modelo de neuroresponsabilidad: una perspectiva de responsabilidad social desde el desarrollo humano. International Journal of Psychological Research, 24-28.

Martí, J., Martí-Vilar, M., \& Almerich,G. (2014). Responsabilidad Social Universitaria: influencia de valores y empatía en la autoatribución de comportamientos socialmente responsables. Revista latinoamericana de Psicología, 46(3), 160-168. https://doi. org/10.1016/S0120-0534(14)70019-6

Martí, J., Moncayo, E., \& Martí-Vilar, M. (2014). Revisión de propuestas metodológicas para evaluar la responsabilidad social universitaria. Revista digital de investigación en docencia universitaria, 8, 78-96.

Martí-Vilar, M., Almerich, G., Cifuentes, J, Grimaldo, M., Martí, J., \& Puerta , I. (2011). Responsabilidad Social Universitaria: Estudio Iberoamericano sobre la influencia de la educación en la formación de profesionales responsables con la sociedad. Revista ciencia y técnica administrativa.

Martí-Vilar, M., Vargas, O, Moncayo, J, \& Martí, J.J. (2014). El modelo de educación superior y el desarrollo del rezonamiento moral. Brazilian Geographical Journal: Geosciences and Humanities research medium, Ituiutaba, 5(2), 398-414. Recuperado Septiembre de 2018, de https://www.researchgate.net/profile/Manuel_Marti_Vilar/publication/268506888_La_for- macion_en_razonamiento_moral_y_pensamiento_ critico_en_la_Educacion_

Montesi, M., \& Villaseñor, I. (2018). Aprendizaje y Servicio (ApS) en los estudios de información y documentación: resultados preliminares de un proyecto UCM. VII seminario Hispano- Brasileño en investigación en información, Documentación y sociedad. . Murcia. Recuperado de https://www.researchgate.net/ publication/328901805

Navarro, G. (2013). Educación: Base para la Responsabilidad Social. Concepción: Universidad de Concepción.

Puig, J., \& Bär, B. (2016). Reconocimiento y aprendizaje y servicio. Revista Iberoamericana de aprendizaje y servicio, 2, 139-165. Recuperado de http://hdl.handle. net/2445/154799

Quinteros, C. (2019). Legitimidad territorial de las universidades chilenas: discusiones y desafíos para la vinculación con el medio. +E: Revista de de Extensión Universitaria, 9(10), 38-59. https://doi.org/10.14409/ extension.v9i10.Ene-Jun.8288

Rodríguez-Martín, A. (2017). Practicas innovadoras inclusivas: retos y oportunidades. Oviedo: Universidad de Oviedo.

Ruiz-Berajano, A., \& Alastor, E. (2018). La organización de jornadas en el aula académica: Innovación docente y compromiso social universitario. Revista Digital de Investigación en Docencia Universitaria, 12(1), 176-188. https://doi.org/10.19083/ridu.12.675

Salazar, E., Salazar, M, Rodríguez, C, \& Díaz, J. (2019). Descripción de actitudes sobre la ética profesional en estudiantes de dos escuelas profesionales en el área de salud. An Fac med, 80(3), 342-5. https://doi. org/10.15381/anales.803.16858

Simó, S. (2013). El aprendizaje servicio universitario: materializando el compromiso social de la universidad desde una educación basada en la excelencia. Estudios sobre el mensaje periodístico, 19, 10271036. Recuperado de https://doi.org/10.5209/rev_ ESMP.2013.v19.42187

Simó, S., Ginesta, X., \& San Eugenio, J. (2013). Aprendizaje servicio Universitario: creando empleo a partir de la emprenduría social. Historia y Comunicación Social, 18, 627-638. https://doi.org/10.5209/rev_HICS.2013. v18.44265

Sosa, M., Espinosa, A., Corne, R, Corne, E, Reyes, M, \& Leal, M. (2016). Acciones para reforzar los valores responsabilidad y humanismo en los futuros profesionales de la salud. Educentro, 8(1), 96-110.

Trujillo, Z. (2016). Experiencia investigativa en docentes 
forjadores de valores profesionales en estudiantes de ciencias médicas. Ciencias Médicas de Pinar de Río, 20(6), 721-728.

Vallaeys, F. (2008). Responsabilidad Social Universitaria: una nueva filosifía de gestión ética e inteligente para las universidades. Revista Educación Superior y Sociedad, 13(2), 191-219.

Valleys, F. (2018). Las diez falacias de la Responsabilidad Social Universitaria. Revista digital de Investigación en Docencia Universitaria, 12(1), 34-58. https://doi.

\section{org/10.19083/ridu.12.716}

Véliz, A., Döner, A., Gonzáles, E., \& Ripoll, M. (2017). Pérfil de valores de estudiantes de carreras de salud en el sur de Chile. Revista Horizonte Médico, 17(2), 48-54. https://doi.org/10.24265/horizmed.2017.v17n2.07

Vera, M., Cornejo, R., Rivas, L., Calizaya, J., Zamora, O., \& García, J. (2016). Significado de los valores profesionales en docentes y estudiantes de enfermería, UNMSM-2014. An Fac Med, 77(3), 225-9. http://doi. org/10.15381/anales.v77i3.12404\

RIDU / Revista Digital de Investigación en Docencia Universitaria / e-ISSN: 2223-2516

(c) Los autores. Este artículo es publicado por la Revista Digital de Investigación en Docencia Universitaria del Área de Institutional Research and Effectiveness de la Dirección de Aseguramiento de la Calidad, Universidad Peruana de Ciencias Aplicadas. Este es un artículo de acceso abierto, distribuido bajo los términos de la LicenciaCreativeCommons Atribución-CompartirIgual 4.0 Internacional.( http://creativecommons.org/licenses/by-sa/4.0/), que permite el uso no comercial, distribución y reproducción en cualquier medio, siempre que la obra original sea debidamente citada. 